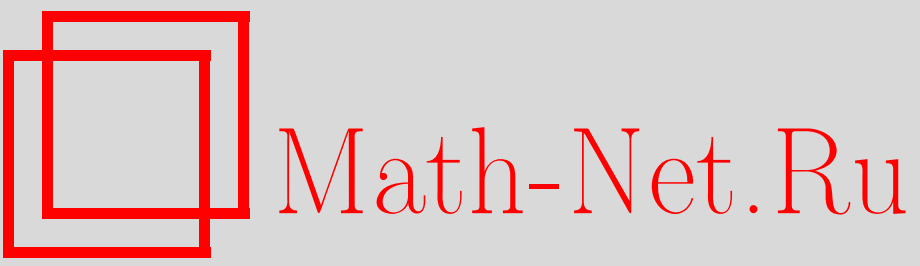

Р. В. Арутюнян, Граничные интегральные уравнения задачи Стефана в терминах времени фазового перехода, Итоги науки и техн. Сер. Соврем. мат. и ее прил. Темат. обз., 2021, том 192, 3-19

DOI: https://doi.org/10.36535/0233-6723-2021-192-3-19

Использование Общероссийского математического портала Math-Net.Ru подразумевает, что вы прочитали и согласны с пользовательским соглашением

http: //www.mathnet.ru/rus/agreement

Параметры загрузки:

IP : 18.207 .199 .55

26 апреля 2023 г., 16:53:53 


\title{
ГРАНИЧНЫЕ ИНТЕГРАЛЬНЫЕ УРАВНЕНИЯ ЗАДАЧИ СТЕФАНА В ТЕРМИНАХ ВРЕМЕНИ ФАЗОВОГО ПЕРЕХОДА
}

\author{
(C) 2021 г. $\quad$ P. В. АРУТЮНЯН
}

\begin{abstract}
АннотАция. В статье рассмотрен новый численный метод для решения задачи Стефана, использующий функцию времени фазового перехода. Осуществлен вывод нелинейного интегрального уравнения минимальной размерности. Описан эффективный численный метод решения интегрального уравнения. Вычислительные качества метода исследованы на примере решения практически важных задач оттаивания мерзлых грунтов при различных способах термического воздействия.
\end{abstract}

Ключевые слова: задача Стефана, интегральное уравнение, численный метод, оттаивание грунтов.

\section{BOUNDARY INTEGRAL EQUATIONS OF THE STEFAN PROBLEM IN TERMS OF THE TIME OF PHASE TRANSITION}

\author{
C 2021 R. V. HARUTYUNYAN
}

\begin{abstract}
In this paper, a new numerical method for solving the Stefan problem based on the time function of phase transitions is considered. A nonlinear integral equation of minimal dimension is obtained and an efficient numerical method for solving this equation is proposed. The method is tested on important problems of thawing of frozen soils with various methods of thermal action.
\end{abstract}

Keywords and phrases: Stefan problem, integral equation, numerical method, soil thawing.

AMS Subject Classification: $80 \mathrm{~A} 22$

1. Введение и постановка задачи Стефана. Моделирование процессов теплопереноса с учетом фазовых превращений является практически важной задачей во многих областях науки и техники (металлургия, электросварка, термообработка материалов, строительство и эксплуатации зданий в условиях зимних температур, технологии хранения и обработки продуктов и т. д.). Соответствующая краевая задача для уравнения теплопроводности называется задачей Стефана и имеет следующий вид:

$$
\begin{gathered}
\rho c \frac{\partial u}{\partial t}=\operatorname{div}(\lambda \operatorname{grad} u)+f(M, t), \quad M \in V, \quad t>0, \\
\lambda^{+} \frac{\partial u^{+}}{\partial n_{f}}-\lambda^{-} \frac{\partial u^{-}}{\partial n_{f}}=\rho r_{\text {melt }} v_{n}, \quad u^{+}=u^{-}=u_{f}, \quad M \in S_{f}, \quad t>0, \\
\lambda \frac{\partial u}{\partial n}+\kappa u=q, \quad M \in S, \quad t>0, \\
u(M, 0)=u_{0}(M), \quad M \in V, \\
|u(M, t)|<\text { const }<\infty,
\end{gathered}
$$


где $V$ - область $m$-мерного евклидова пространства $E^{m}$, не имеющая разрезов, с кусочно гладкой границей $S=\partial V, u$ - функция, описывающая нестационарное температурное поле при наличии фазовых превращений на поверхности $S_{f}$, определяемой соответствующими условиями (1)-(5), $u_{f}$ - температура фазового перехода, индекс «-» соответствует области жидкой, а индекс «+»- области твердой фазы, $u_{0}(M)$ - начальное распределение температур в области $V$, $\partial u^{ \pm} / \partial n_{f}=\nabla u\left(M^{ \pm}, t\right) \boldsymbol{n}_{f}(M, t)$, где $\boldsymbol{n}_{f}(M, t)$ - вектор единичной нормали в точке $M$ поверхности $S_{f}$, в момент времени $t$, внешней по отношению к области $V_{f}: V_{f}=\left\{M \in V \mid u(M, t)>u_{f}\right\}$,

$$
\begin{gathered}
\nabla u\left(M^{-}, t\right)=\lim _{N \rightarrow M ; N \in V_{\text {melt }}} u(N, t), \quad M \in S_{f}, \quad t>0, \\
\nabla u\left(M^{+}, t\right)=\lim _{N \rightarrow M ; N \in V-V_{\text {melt }}} u(N, t), \quad M \in S_{f}, \quad t>0, \\
\frac{\partial u^{ \pm}}{\partial n_{f}}=\nabla u\left(M^{ \pm}, t\right) \boldsymbol{n}_{f}(M, t), \quad v_{n}(M, t)=\boldsymbol{v}_{f}(M, t) \boldsymbol{n}_{f}(M, t),
\end{gathered}
$$

где $\boldsymbol{v}_{f}(M, t)$ - вектор скорости движения поверхности $S_{f}$ в точке $M \in S_{f}$ в момент времени $t>0$.

Функции $\rho(M), c(M), \lambda(M), f(M, t), r_{\text {melt }}(M), \kappa(M)$ будем считать таковыми, что существует классическое решение задачи $(1)-(5) u(M, t)$ и классическое решение задачи $(1)-(5)$ при $r_{\text {melt }}=$ 0 , которое обозначим $H(M, t)$. Согласно [2-6,8-10] это будет выполнено, например, если $\rho(M)$, $c(M), \lambda(M), f(M, t), r_{\text {melt }}(M), \kappa(M)$ будут положительными константами, $f \in L_{2}(V), q \in L_{2}(S)$, $u_{0}(M) \in C^{1}(V)$.

При $v_{n}(M, t) \geqslant 0$ выполнится условие

$$
V_{f}\left(t_{1}\right) \subseteq V_{f}\left(t_{2}\right), \quad 0<t_{1}<t_{2}<\vartheta,
$$

где $0<t<\vartheta$-интервал времени, на котором происходит расширение области плавления. Задача (1)-(5) приводится к эквивалентному виду с так называемой сосредоточенной теплоемкостью, учитывающей теплоту фазового перехода (см. $[2,9])$ :

$$
\begin{gathered}
\rho\left[c+r_{\text {melt }} \delta\left(u-u_{f}\right)\right] \frac{\partial u}{\partial t}=\operatorname{div}(\lambda \nabla u)+f(M, t), \quad M \in V, \quad t>0, \\
\lambda \frac{\partial u}{\partial n}+\kappa u=q, \quad M \in S, \quad t>0, \\
u(M, 0)=u_{0}(M), \quad|u(M, t)|<\text { const }<\infty, \quad M \in V,
\end{gathered}
$$

где $\delta(x)$ - дельта-функция Дирака.

\section{2. Редукция краевой задачи к интегральному уравнению}

минимальной размерности. Представим $u(M, t)$ как сумму $u=w+H$, где $H$-решение краевой задачи без учета стефановской нелинейности. Можно показать, что $w$ удовлетворяет следующим условиям:

$$
\begin{gathered}
\rho c w+r_{\text {melt }}\left[1\left(w+H-u_{f}\right)-1\left(u_{0}-u_{f}\right)\right]=\operatorname{div}(\lambda \nabla W), \quad M \in V, \quad t>0, \\
\lambda \frac{\partial w}{\partial n}+\kappa w=0, \quad M \in S, \quad t>0, \\
w(M, 0)=0, \quad|w(M, t)|<\text { const }<\infty, \quad M \in V,
\end{gathered}
$$

где $1(x)$ - единичная функция Хевисайда,

$$
W(M, t)=\int_{0}^{t} w(M, \tau) d \tau .
$$

Пусть $G(M, N, t)$ - функция Грина линейной краевой задачи, получающейся из (10)-(12) при $r_{\text {melt }}=0$. Тогда решение (10)-(12) удовлетворяет уравнению

$$
W(M, t)=\int_{0}^{t} \int_{V} \rho(N) r_{\text {melt }}(N) G(M, N, t-\tau)\left[1\left(u_{0}(N)-u_{f}\right)-1\left(u(N, \tau)-u_{f}\right)\right] d V_{N} d \tau,
$$




$$
M \in V+S, \quad t>0 .
$$

Продифференцировав последнее равенство по времени, получим (с учетом известных свойств функции Грина):

$$
\begin{aligned}
w(M, t) & =\rho r_{\text {melt }}\left[1\left(u_{0}-u_{f}\right)-1\left(u-u_{f}\right)\right]+ \\
& +\int_{0}^{t} \int_{V} \rho(N) r_{\text {melt }}(N) \frac{\partial G}{\partial t}(M, N, t-\tau)\left[1\left(u_{0}(N)-u_{f}\right)-1\left(u(N, \tau)-u_{f}\right)\right] d V_{N} d \tau, \\
& M \in V+S, \quad t>0 .
\end{aligned}
$$

Введем в рассмотрение функцию $t_{f}(M)$, являющуюся наименьшим корнем уравнения $u\left(M, t_{f}(M)+0\right)=u_{f}+0, M \in V+S$. В точках $M \in V+S$, где такого решения не существует, положим значение $t_{f}(M)$ бесконечным. Функция $t_{f}(M)$ имеет смысл времени фазового превращения вещества в точке $M$ (в рассматриваемом случае из твердого в жидкое). В силу определения $t_{f}(M)$ и при выполнении (6) имеем

$$
V_{f}(t)=\left\{M \in V \mid t_{f}(M)<t\right\}, \quad t<\vartheta
$$

откуда

$$
S_{f}(t)=\left\{M \in V \mid t_{f}(M)=t\right\} . \quad t<\vartheta,
$$

Так как для всех $t<\vartheta, M \in V+S$ имеем $1\left(u(M, t)-u_{f}\right)=1\left(t-t_{f}(M)\right)$, то (13) преобразуется к виду

$$
w(M, t)=-\int_{V} \rho(N) r_{\text {melt }}(N) G\left(M, N, t-t_{f}(N)\right) 1\left(t-t_{f}(N)\right) d V_{N}, \quad M \in V+S, \quad t<\vartheta .
$$

Учитывая, что $w(M, t)=u_{f}-H\left(M, t_{f}(M)\right)$ при $t=t_{f}(M)$, получаем из (14) нелинейное интегральное уравнение Фредгольма второго рода относительно функции $t_{f}(M)$ :

$$
\begin{gathered}
\int_{V} \rho(N) r_{\text {melt }}(N) G\left(M, N, t_{f}(M)-t_{f}(N)\right) 1\left(t_{f}(M)-t_{f}(N)\right) d V_{N}=H\left(M, t_{f}(M)\right)-u_{f}, \\
M \in V+S .
\end{gathered}
$$

3. Исследование интегрального уравнения. Существование кусочно непрерывного решения уравнения (15) следует из его вывода. Для доказательства единственности любого кусочно непрерывного решения (15) в области $V_{f}(\vartheta)$ покажем, что справедливо

Утверждение 1. Пусть $z(M)$ - кусочно непрерывное решение уравнения (15), удовлетворяющее следуюшим условиям:

(1) $z(M)<\vartheta$ для всех $M \in V_{0} \subset V$;

(2) вектор $\boldsymbol{n}_{0}=\nabla z(M) /|\nabla z(M)|$ является вектором внешней единичной нормали к границе области $\Omega=\{M \in V \mid z(M)<t\}, t<\vartheta ;$ тогда $z(M)=t_{f}(M)$ для всех $M \in V_{f}(\vartheta), V_{0}=V$.

Доказательство. Имеем

$$
R(M, \tau)=H(M, \tau)-\int_{V} \rho(N) r_{\text {melt }}(N) G(M, N, \tau-z(N)) 1(\tau-z(N)) d V_{N}, \quad M \in V+S .
$$

Поскольку $z(M)$ - решение (15), то $R(M, z(M))=u_{f}, M \in V_{0}$. Проведя преобразования, обратные осуществленным при выводе (15), получим, что функция $R(M, \tau)$ необходимым образом является решением краевой задачи

$$
\begin{gathered}
\rho c R+\rho r_{\text {melt }} \delta(\tau-z(M))=\operatorname{div}(\lambda \nabla R)+f(M, \tau), \quad M \in V, \quad \tau>0, \\
\lambda \frac{\partial R}{\partial n}+\kappa R=q, \quad M \in S, \quad \tau>0, \\
R(M, 0)=0, \quad|R(M, \tau)|<\text { const }<\infty, \quad M \in V,
\end{gathered}
$$




$$
R(M, z(M))=u_{f}, \quad M \in V_{0},
$$

откуда

$$
R(M, \tau)=H(M, \tau)-\int_{0}^{\tau} \int_{V} \rho(N) r_{\text {melt }}(N) G(M, N, \tau-\xi) \delta(\xi-z(N)) d V_{N} d \xi, \quad M \in V+S, \quad \tau>0 .
$$

Учитывая известное тождество

$$
\frac{d}{d \tau} \int_{\omega(\tau)} f(N, \tau) d V_{N}=\int_{\partial \omega(\tau)} f(N, \tau) v_{n}(N, \tau) d S_{N}+\int_{\omega(\tau)} \partial f(N, \tau) / \partial \tau d V_{N}
$$

где $v_{n}(N, \tau)$ - нормальная составляющая вектора скорости движения границы области $\omega(\tau)$ в точке $N$ в момент времени $\tau$ (внешняя нормаль к $\omega(\tau))$, находим, что

$$
R(M, \tau)=H(M, \tau)-\int_{0}^{\tau} \int_{S_{z}(\tau)} \rho(N) r_{\text {melt }}(N) G(M, N, \tau-\xi) v_{n}(N, \xi) d S_{N} d \xi, \quad M \in V+S,
$$

где $S_{z}(\tau)=\partial \omega(\tau), \omega(\tau)=\{M \in V \mid z(M)<\tau\}$; так как $z(M)=\tau$ на поверхности $S_{z}(\tau)$, то $R(M, \tau)=u_{f}, M \in S_{z}(\tau) \subset V_{0}, \tau<\vartheta$. Учитывая известные свойства теплового потенциала простого слоя (каковым является второе слагаемое в $(20))$ и непрерывную дифференцируемость $H(M, \tau)$, получаем, что при переходе через поверхность $S_{z}(\tau)$ нормальная производная от $R(M, \tau)$ терпит скачок первого рода:

$$
\lambda \frac{\partial R^{+}}{\partial n_{z}}-\lambda \frac{\partial R^{-}}{\partial n_{z}}=r_{\text {melt }} v_{n}, \quad M \in S_{z}(\tau)
$$

где $\boldsymbol{n}_{z}$ - вектор единичной нормали к $S_{z}(\tau)$, внешней по отношению к $\omega(\tau)$.

Равенства (16)-(22) означают, что для $\tau<\vartheta$ функция $R(M, \tau)$ удовлетворяет задаче Стефана (1)-(5) и в силу единственности ее решения $R(M, \tau)=u(M, \tau), M \in V+S, \tau<\vartheta$. Поскольку наименьший корень уравнения $u(M, \tau+0)=u_{f}+0, M \in V+S$, единственный, то $z(M)=t_{f}(M)$ для всех $M \in V_{f}(\vartheta)$.

\section{4. Методы численного решения нелинейного интегрального уравнения (15).}

4.1. Метод ячеек. Осуществим разбиение области $V$ на элементы $V_{j}, j=1, \ldots, N(N$ может быть бесконечным),

$$
V=\bigcup_{j=1}^{N} V_{j}, \quad V_{i} \cap V_{j}=\varnothing, \quad i \neq j
$$

В пределах $V_{j}$ считаем, что $t_{f}(M) \approx t_{j}=$ const, $\rho(M) r_{\text {melt }}(M) \approx \rho_{j} r_{\text {melt,j, }}, H(M, t) \approx H_{j}(t)$. Тогда получаем следующую систему нелинейных уравнений относительно неизвестных $t_{1}, \ldots, t_{N}$ :

$$
\begin{gathered}
\sum_{j=1}^{N} \frac{r_{\text {melt }, j}}{c_{j}} x_{i j}\left(t_{i}-t_{j}\right) 1\left(t_{i}-t_{j}\right)=H_{i}\left(t_{i}\right)-u_{f}, \quad i=1, \ldots, N ; \\
x_{i j}(t)=\int_{V_{j}} \rho_{j} c_{j} G\left(M_{i}, N, t\right) d V_{N}, \quad M_{i} \in V_{i},
\end{gathered}
$$

где $M_{i}$ - узел коллокации, как правило, находящийся в центре ячейки $V_{i}$.

Построим конструктивное доказательство возможности построения для задачи (1)-(5) корректного приближенного метода, основанного решении на системы вида

$$
\sum_{j=1}^{N} r_{\text {melt }, j} c_{j} y_{i j}\left(t_{i}-t_{j}\right) 1\left(t_{i}-t_{j}\right)=H_{i}\left(t_{i}\right)-u_{f}, \quad i=1, \ldots, N
$$


Для этого введем в рассмотрение схему метода прямых, аппроксимирующую (10)-(12):

$$
\begin{gathered}
\rho_{i} c_{i} w_{i}=\left(A^{h} W^{h}\right)_{i}+\rho_{i} r_{\text {melt }, i}\left[1\left(u_{0 i}-u_{f}\right)-1\left(u_{i}-u_{f}\right)\right], \quad i \in V^{h}, \\
B^{h}\left(w_{j}^{h}\right)=0, \quad j \in S_{h}=\partial V^{h},
\end{gathered}
$$

где $V^{h}$-сетка с узлами $M_{1}, \ldots, M_{N}$, покрывающая $V, w_{i}$-компоненты вектор-функции $w^{h}$, $A^{h}$ - конечно-разностный оператор, аппроксимирующий дифференциальный оператор $\operatorname{div}(\lambda \nabla \cdot)$, $A^{h} \geqslant 0$, причем на функциях, удовлетворяющих однородному краевому условию в (24):

$$
u_{i}=w_{i}+H\left(M_{i}, t\right), \quad u_{0 i}=u_{0}\left(M_{i}\right) .
$$

Возможность построения такой схемы прямых следует из известных результатов (см. [4]). Решения (24) удовлетворяют условиям

$$
\left\|w^{h}\right\|_{l_{2}\left(V^{h}\right)}<c_{0}=\text { const }
$$

при

$$
h=\max _{i \in V^{h}} \min _{j \in\left\{V^{h}-i\right\}}\left|M_{i} M_{j}\right| \rightarrow 0, \quad\left\|w^{h}-P^{h} w\right\|_{l_{2}\left(V^{h}\right)} \rightarrow 0
$$

где $P^{h}$-оператор сужения, $P^{h} w=\left(w\left(M_{1}, t\right), \ldots, w\left(M_{N}, t\right)\right)$,

$$
\left\|w^{h}\right\|_{l_{2}\left(V^{h}\right)}=\left(\int_{0}^{\vartheta} \sum_{j \in V^{h}} w_{j}^{2}(t)\left|V_{j}\right| d t\right)^{1 / 2}, \quad\left|V_{j}\right|=\operatorname{mes} V_{j} .
$$

Для равномерной сетки $\left|V_{j}\right|=h^{m}$. Соотношения (24) перепишем в виде

$$
w^{h}=\widetilde{A}^{h} W^{h}+\widetilde{F}^{h}, \quad \widetilde{F}_{i}^{h}=\frac{r_{\mathrm{melt}, i}}{c_{i}}\left[1\left(u_{0 i}-u_{f}\right)-1\left(u_{i}-u_{f}\right)\right], \quad i \in V^{h},
$$

где $\left\{\widetilde{A}^{h} W^{h}\right\}_{i}=\left\{A^{h} W^{h}\right\}_{i} /\left(\rho_{i} c_{i}\right)$.

Пусть $y_{i j}(t)$ - коэффициенты нормальной фундаментальной матрицы $Y[t]$, удовлетворяющей матричной задаче Коши:

где $I$ - единичная матрица:

$$
\dot{Y}=\widetilde{A}^{h} Y, \quad Y[0]=I,
$$

По формуле Коши имеем

$$
\dot{Y}[t]=\left\|\frac{d y_{i j}}{d t}\right\|_{j=1, \ldots, N}^{i=1, \ldots, N}
$$

$$
W^{h}[t]=\int_{0}^{t} Y[t-\tau] \widetilde{F}^{h}[\tau] d \tau
$$

После дифференцирования получаем

$$
\begin{aligned}
w_{i}(t)=\frac{r_{\mathrm{melt}, i}}{c_{i}}\left[1\left(u_{0 i}-u_{f}\right)\right. & \left.-1\left(u_{i}-u_{f}\right)\right]+ \\
+ & \sum_{j \in V^{h}} \frac{r_{\mathrm{melt}, j}}{c_{j}} \int_{0}^{t} \dot{y}_{i j}(t-\tau)\left[1\left(u_{j}(\tau)-u_{f}\right)-1\left(u_{0 j}-u_{f}\right)\right] d \tau, \quad i \in V^{h} .
\end{aligned}
$$

Введем в рассмотрение вектор $t^{h}$, соответствующий выше определенной функции $t_{f}(M): t^{h}=$ $\left(t_{1}, \ldots, t_{N}\right)$, где $t_{i}$ - наименьший неотрицательный корень уравнения $u_{i}(t+0)=u_{f}+0, t>0$. Если для какого-то $i$ такого корня не существует, то полагаем $t_{i}=\infty$. Тогда $(26)$ переписываются в виде:

$$
w_{i}(t)=-\sum_{j \in V^{h}} r_{\mathrm{melt}, j} c_{j} y_{i j}\left(t-t_{j}\right) 1\left(t-t_{j}\right), \quad i \in V^{h}, \quad t<\vartheta^{h}
$$

где $\left[0, \vartheta^{h}\right]$ - интервал времени, для которого выполнено свойство:

$$
V_{f}^{h}\left(t_{1}\right) \subseteq V_{f}^{h}\left(t_{2}\right), \quad 0<t_{1} \leqslant t_{2}<\vartheta^{h} .
$$


$V_{f}^{h}(t)=\left\{i \in V^{h} \mid u_{i}(t)>u_{f}\right\}$. При $t=t_{i}(27)$ обращается в систему нелинейных уравнений относительно $t^{h}$ :

$$
\sum_{j \in V^{h}} r_{\mathrm{melt}, j} c_{j} y_{i j}\left(t_{i}-t_{j}\right) 1\left(t_{i}-t_{j}\right)=H_{i}\left(t_{i}\right)-u_{f}, \quad i \in V^{h}
$$

4.2. Исследование численного метода. Существование и единственность решения системы уравнений (29) в области $V_{f}^{h}\left(\vartheta^{h}\right)$ показывается аналогично тому, как это было сделано ранее для функции $t_{f}(M)$. Сходимость $t^{h}$ к $t_{f}(M)$ оценивается ниже.

Утверждение 2. Пусть существуют такие не зависящие от $h<h_{0}$ числа $\gamma>0, \vartheta_{1}>0$, $k>0$, чmо $\vartheta_{1}<\min \left\{\vartheta, \vartheta^{h}\right\}, k=k\left(\vartheta_{1}, \gamma\right)$,

$$
\sum_{j \in v_{\vartheta_{1}}}\left(\int_{t_{j}}^{\vartheta_{1}} \frac{d \tau}{\left|u_{j}(\tau)-u_{f}\right|^{\gamma}}+\int_{t_{f}\left(M_{j}\right)}^{\vartheta_{1}} \frac{d \tau}{\left|u\left(M_{j}, \tau\right)-u_{f}\right|^{\gamma}}\right)\left|V_{j}\right|<K,
$$

где подмножество $v_{\vartheta_{1}} \subseteq V^{h}$ определяется из условия

$$
\max _{i \in \vartheta_{\vartheta_{1}}}\left\{t_{i}, t_{f}\left(M_{i}\right)\right\}<\vartheta_{1} .
$$

Тогда

$$
\lim _{h \rightarrow 0}\left\|t^{h}-P^{h} t_{f}\right\|_{l_{p}\left(v_{\vartheta_{1}}\right)}=0, \quad p \geqslant 1 .
$$

Доказательство. Поскольку

$$
\int_{0}^{t} 1\left(\tau-t_{i}\right) d \tau=\left(t-t_{i}\right) 1\left(t-t_{i}\right)
$$

следовательно, при $i \in v_{\vartheta_{1}}$ имеем

$$
t_{f}\left(M_{i}\right)-t_{i}=\int_{0}^{\vartheta_{1}}\left[1\left(u_{i}(\tau)-u_{f}\right)-1\left(u\left(M_{i}, \tau\right)-u_{f}\right)\right] d \tau
$$

откуда

$$
\left|t_{f}\left(M_{i}\right)-t_{i}\right|^{p}=\left(\int_{0}^{\vartheta_{1}}\left|1\left(u_{i}(\tau)-u_{f}\right)-1\left(u\left(M_{i}, \tau\right)-u_{f}\right)\right| d \tau\right)^{p}, \quad p \geqslant 1 .
$$

В силу неравенства

$$
\left(\frac{1}{\vartheta_{1}} \int_{0}^{\vartheta_{1}}\left|1\left(u_{i}(\tau)-u_{f}\right)-1\left(u\left(M_{i}, \tau\right)-u_{f}\right)\right| d \tau\right)^{p} \leqslant \frac{1}{\vartheta_{1}} \int_{0}^{\vartheta_{1}}\left|1\left(u_{i}(\tau)-u_{f}\right)-1\left(u\left(M_{i}, \tau\right)-u_{f}\right)\right| d \tau
$$

получаем, что

$$
\left\|t^{h}-P^{h} t_{f}\right\|_{l_{p}\left(v_{\vartheta_{1}}\right.} \leqslant\left\|t^{h}-P^{h} t_{f}\right\|_{l_{1}\left(v_{(} \vartheta_{1}\right)}^{1 / p}, \quad p \geqslant 1 .
$$

В силу свойства единичной функции

$$
|1(x+y)-1(x)| \leqslant\left|\frac{y}{x}\right|^{z}, \quad z>0, \quad\{x, y\} \subset E^{1},
$$

имеем

$$
\left|t_{f}\left(M_{i}\right)-t_{i}\right| \leqslant\left.\left.\left|\int_{t_{i}}^{t_{f}\left(M_{i}\right)}\right| \frac{u_{i}(\tau)-u\left(M_{i}, \tau\right)}{u_{i}(\tau)-u_{f}}\right|^{b} d \tau\left|+\int_{t_{f}\left(M_{i}\right)}^{\vartheta_{1}}\right| \frac{u_{i}(\tau)-u\left(M_{i}, \tau\right)}{u\left(M_{i}, \tau\right)-u_{f}}\right|^{b} d \tau .
$$

Следовательно, с учетом неравенства Коши-Буняковского, получаем неравенство 


$$
\begin{aligned}
& \| t^{h}- P^{h} t_{f} \|_{l_{1}\left(v_{\vartheta_{1}}\right)} \leqslant \\
& \leqslant\left(\sum_{j \in v_{\vartheta_{1}}} \int_{t_{f}\left(M_{j}\right)}^{\vartheta_{1}}\left|u_{j}(\tau)-u\left(M_{j}, \tau\right)\right|^{b s} d \tau\left|V_{j}\right|\right)^{1 / s}\left(\sum_{j \in \vartheta_{\vartheta_{1}}} \int_{t_{f}\left(M_{j}\right)}^{\vartheta_{1}}\left|u\left(M_{j}, \tau\right)-u_{f}\right|^{-b q} d \tau\left|V_{j}\right|\right)^{1 / q}+ \\
&+\left(\sum_{j \in v_{\vartheta_{1}}} \int_{t_{j}}^{t_{f}\left(M_{j}\right)}\left|u_{j}(\tau)-u\left(M_{j}, \tau\right)\right|^{b s} d \tau\left|V_{j}\right|\right)^{1 / s}\left(\sum_{j \in v_{\vartheta_{1}}} \int_{t_{j}}^{t_{f}\left(M_{j}\right)}\left|u_{j}(\tau)-u_{f}\right|^{-b q} d \tau\left|V_{j}\right|\right)^{1 / q}, \\
& s, q>0, \quad \frac{1}{s}+\frac{1}{q}=1 .
\end{aligned}
$$

Выбираем $r q=\gamma, r=\gamma /(1+\gamma / 2)$, тогда $s=1+2 / \gamma, r s=2$,

$$
\left\|t^{h}-P^{h} t_{f}\right\|_{l_{p}\left(v_{\vartheta_{1}}\right)} \leqslant K^{2 /(2+\gamma)}\left\|u^{h}-P^{h} u\right\|_{l_{2}\left(V_{h}\right)}^{c_{0} / p}, \quad c_{0}=\frac{1}{s},
$$

а так как $u^{h}-P^{h} u=w^{h}-P^{h} w$ и при $h \rightarrow 0:\left\|w^{h}-P^{h} w\right\|_{l_{2}\left(V_{h}\right)} \rightarrow 0$, то утверждение доказано.

Условие утверждения 2 упрощается, если не рассматривать однофазные задачи Стефана. Тогда постоянная $K$ может быть выбрана из условия. Оценка величины $\left\|t^{h}-P^{h} t_{f}\right\|_{l_{p}\left(v_{\vartheta_{1}}\right)}$ по форме остается прежней.

5. Алгоритмы решения систем нелинейных уравнений (22) и (29). Если размерность систем $(22)$ и (29) бесконечна, число непересекающихся связных подмножеств $V_{f}^{h}(t)$ конечно и не возрастает во времени, то эффективным может быть следующий метод решения. Исходным является следующее свойство:

$$
\forall i \in V_{f}^{h}, j \in \partial V_{f}^{h}: \quad t_{i}<t_{j}, \quad 1\left(t_{i}-t_{j}\right)=0 .
$$

Обозначим $V_{f}^{h}(t)$ для краткости через $\sigma$; тогда алгоритм примет следующий вид.

1. (а) Задаем конечное число $t_{\max }$.

(b) Определяем начальное множество узлов $\sigma=\left\{i_{1}, \ldots, i_{l}\right\}$, для которых $H_{i_{1}}\left(t_{i_{1}}\right)=\ldots=$ $H_{i_{l}}\left(t_{i_{l}}\right)=u_{f}, t_{i_{1}}=\ldots=t_{i_{l}}=z_{*}$.

(c) Задаем число $\varepsilon$. Если компоненты вектора $t^{h}$ различаются менее, чем на $\varepsilon$, то они считаются равными.

2. Формируем множество узлов $\partial \sigma$, граничных к $\sigma$.

3. Определяем размерность $\partial \sigma: n=\operatorname{dim} \partial \sigma, \partial \sigma=\left\{j_{1}, \ldots, j_{n}\right\}$.

4. Присваиваем $p$ значение, равное 1.

5. Полагаем $z_{\min }=z_{*}$.

6. Вычисляем $z_{\max }=t_{\max }, p=1 ; z_{\max }=\min \left\{t_{\max }, z_{1}, \ldots, z_{p-1}\right\}, p>1$.

7. Находим корень $z_{p}$ уравнения

$$
H_{j_{p}}\left(z_{p}\right)-u_{f}-\sum_{j \in \sigma} \frac{r_{\mathrm{melt}, j}}{c_{j}} x_{j_{p} j}\left(z_{p}-t_{j}\right) 1\left(z_{p}-t_{j}\right)=0,
$$

лежащий в интервале $z_{\min } \leqslant z_{p} \leqslant z_{\max }, j_{p} \in \partial \sigma$.

8. Увеличиваем значение $p$ на единицу.

9. Проверяем, не исчерпаны ли элементы $\partial \sigma$, что равносильно неравенству $p>n$. Если да, то переходим к п. 12, иначе к п. 6.

10. Полагаем $z_{*}=\min _{j_{p} \in \partial \sigma} z_{p}$.

11. Проверяем выполнение неравенства $z_{*} \geqslant t_{\max }$. Если оно справедливо, то вычисления завершены, иначе переходим к п. 12.

12. Формируем множество узлов $\omega=\left\{j_{p} \in \partial \sigma:\left|z_{p}-z_{*}\right| \leqslant\right\}$.

13. Расширяем множество $\sigma$, присоединяя к нему множество $\omega$.

14. Переходим к п. 3. 
Из рассмотрения описанного метода следует, что в нем строится неубывающая последовательность значений компонент вектора решения системы $(29) t^{h}: t_{i_{1}} \leqslant \ldots t_{i_{n}} \leqslant \ldots$ Таким образом, разыскиваемое решение автоматически удовлетворяет условию: $t_{i} \leqslant \vartheta^{h}$, хотя значение $\vartheta$ априорно неизвестно. Существенно, что изложенный алгоритм легко адаптируется для программирования на многопроцессорных компьютерах. Распараллеливанию подлежит легко расщепляющийся, но наиболее трудоемкий п. 7. Между процессорами следует распределить связные непересекающиеся между собой подмножества $\partial \sigma$, с размерностями, пропорциональными быстродействию соответствующего процессора. Разбиение целесообразно осуществлять таким образом, чтобы диаметр каждого подмножества стремился к нулю при увеличении числа процессоров.

6. Вычисление сингулярного слагаемого в суммах (22) и (29). Слагаемые $x_{i j}\left(t-t_{j}\right)$, $y_{i j}\left(t-t_{j}\right)$ при $i=j$ являются сингулярными, так как функция $G\left(M_{i}, M_{i}, t\right)$ неограниченна в окрестности нуля $t$. При этом вклад данного слагаемого относительно значителен. По этим причинам существует целесообразность вычисления сингулярного слагаемого аналитическими методами. Для этого в соответствующей ячейке $V_{i}$ функция $t_{f}(M)$ аппроксимируется линейной частью ряда Тейлора: $t_{f}(M) \approx t_{f}\left(M_{i}\right)+\boldsymbol{k} \cdot \overrightarrow{M M_{i}}$. Во многих случаях на начальном этапе вычислений желательна квадратичная аппроксимация функции $t_{f}(M)$, так как ее рельеф часто имеет параболический вид с центром в начале координат. Рассмотрим вычисление сингулярного слагаемого в одномерном случае (для краткости изложения считаем размер ячеек постоянным):

$$
y_{i i}=\int_{x_{i}-h / 2}^{x_{i}+h / 2} G_{0}\left(x_{i}, x, t_{f}(x)-t_{f}\left(x_{i}\right)\right) 1\left(t_{f}(x)-t_{f}\left(x_{i}\right)\right) d x, \quad x \in\left[x_{i}-h / 2, x_{i}+h / 2\right],
$$

где (согласно асимптотике для области сингулярности)

$$
G_{0}(M, N, t) \approx(2 \sqrt{\pi a t})^{-m} \exp \left(-\frac{r_{M N}^{2}}{4 a t}\right) .
$$

Для одномерного случая: $t_{f}(x) \approx t_{f}\left(x_{i}\right)+k\left(x-x_{i}\right)$,

$$
\begin{aligned}
y_{i i} \approx \int_{x_{i}-h / 2}^{x_{i}+h / 2} \frac{\exp \left\{-\frac{\left(x-x_{i}\right)^{2}}{4 a k\left(x-x_{i}\right)}\right\}}{2 \sqrt{\pi a k\left(x-x_{i}\right)}} & 1\left[k\left(x-x_{i}\right)\right] d x= \\
& =\int_{0}^{h / 2} \frac{e^{-x^{2} /(4 a k)}}{2 \sqrt{\pi a k x}} d x=\operatorname{erf}\left(\sqrt{\frac{h}{8 a k}}\right)=\sqrt{\frac{h}{2 \pi a k}}(1+o(1)), \quad h \rightarrow 0 .
\end{aligned}
$$

При практических вычислениях можно использовать для оценки значения $k$ конечно-разностную аппроксимацию производных: $k \approx\left[t_{f}\left(x_{i}\right)-t_{f}\left(x_{i-1}\right)\right] / h$.

7. Обобщение метода на случай немонотонного движения фронта фазового перехода. При произвольном характере движения фронта фазового перехода, когда на рассматриваемом интервале времени происходят переходы в прежнее агрегатное состояние, имеет место равенство:

$$
1\left(u-u_{f}\right)=1\left(t-t_{f 1}^{+}(M)\right)-1\left(t-t_{f 1}^{-}(M)\right)+1\left(t-t_{f 2}^{+}(M)\right)-1\left(t-t_{f 2}^{-}(M)\right)+\ldots,
$$

где $t_{f i}^{+}(M)$ - момент времени $i$-го перехода в точке $M$ из твердой в жидкую фазу, $t_{f i}^{-}(M)$ - момент времени $i$-го перехода в точке $M$ из жидкой фазы в твердую, причем

$$
t_{f i}^{+}(M)<t_{f i}^{-}(M), \quad t_{f i}^{+}(M) \leqslant t_{f, i+1}^{+}(M), \quad i=1, \ldots, n .
$$

Учитывая, что при $t=t_{f i}^{ \pm}(M), u=u_{f}$, находим соответствующую систему интегральных уравнений относительно $t_{f i}^{ \pm}(M)$. Например, при $n=2$ имеем систему 


$$
\begin{aligned}
\int_{V} \rho(N) r_{\text {melt }}(N)\left(G_{+}\left(M, N, t_{f}^{+}(M)-t_{f}^{+}(N)\right)-G_{+}\left(M, N, t_{f}^{+}(M)-t_{f}^{-}(N)\right)\right) d V_{N}= & \\
=H\left(M, t_{f}^{+}(M)\right)-u_{f}, & M \in V, \\
\int_{V} \rho(N) r_{\text {melt }}(N)\left(G_{+}\left(M, N, t_{f}^{-}(M)-t_{f}^{+}(N)\right)-G_{+}\left(M, N, t_{f}^{-}(M)-t_{f}^{-}(N)\right)\right) d V_{N}= & \\
=H\left(M, t_{f}^{-}(M)\right)-u_{f}, \quad & M \in V,
\end{aligned}
$$

где $G_{+}(M, N, t)=G(M, N, t) 1(t)$.

8. Основные преимущества и недостатки метода. Главным преимуществом описанного метода решения задачи Стефана является экономия машинной памяти, поскольку на каждом этапе вычислений запоминаются только найденные на предыдущем шаге компоненты вектора $t^{h}$. Далее, при использовании MKP возникает система уравнений размерности $\operatorname{dim} V^{h}$ для каждого временного слоя, в то время как в описанном методе рассматриваются только узлы области фазового перехода $V_{f}^{h}$, как правило, $\operatorname{dim} V_{f}^{h} \ll \operatorname{dim} V^{h}$. Интегральное уравнение, получаемое в описанном методе, имеет наименьшую из возможных размерность: позволяет снизить размерность задачи на единицу. По сравнению с классическим вариантом метода тепловых потенциалов в 3 раза уменьшается количество неизвестных функций и решаемых интегральных уравнений. Поэтому метод может достичь экономии машинной памяти и времени вычислений. В случае неограниченной области $V$, в отличие от MKP нет необходимости усечения системы уравнений, возможно имеющих бесконечную размерность. Изложенная процедура также позволяет от сингулярностей исходной постановки краевой задачи, осуществлять уточнение решения посредством экстраполяции по Ричардсону при условии гладкости решения (в случае МКР такой возможности нет, так как первые производные решения краевой задачи разрывны). Метод предоставляет также дополнительные удобства при решении обратных задач Стефана, в частности, проблемы управления фронтом фазового перехода. Недостатком метода является трудоемкость построения функции Грина в случае переменных свойств среды. Тем не менее существует широкий класс задач моделирования фазовых переходов, решение которых может быть успешно найдено описанным методом нелинейных интегральных уравнений.

\section{9. Примеры практического применения описанного метода.}

9.1. Задача Коши в $E^{m}$. При постоянных $\lambda, \rho, c$ имеем

$$
\begin{gathered}
G_{0}(M, N, t)=(2 \sqrt{\pi a t})^{-m} \exp \left(-\frac{r_{M N}^{2}}{4 a t}\right), \quad a=\frac{\lambda}{\rho c}, \\
H(M, t)=\int_{E^{m}} u_{0}(N) G_{0}(M, N, t) d V_{N}+\int_{0}^{t} \int_{E^{m}} \frac{f(N, \tau)}{\rho c} G_{0}(M, N, t-\tau) d V_{N} d \tau .
\end{gathered}
$$

Для метода ячеек (23), (24) компоненты матрицы $X[t]$ равны

$$
\begin{gathered}
x_{k_{1} \ldots k_{m}}^{l_{1} \ldots l_{m}}(t)=u_{k_{1}}^{l_{1}}(t) \ldots u_{k_{m}}^{l_{m}}(t), \\
u_{k}^{l}(t)=\frac{1}{2}\left\{\operatorname{erf}\left[\frac{h(k-l+1 / 2)}{2 \sqrt{a t}}\right]-\operatorname{erf}\left[\frac{h(k-l-1 / 2)}{2 \sqrt{a t}}\right]\right\} .
\end{gathered}
$$

Другое допустимое выражение для соответствующей функции:

$$
u_{k}^{l}(t)=I_{k-l}\left(\frac{2 a t}{h^{2}}\right) \exp \left(-\frac{2 a t}{h^{2}}\right),
$$

примечательно тем, что является точным решением системы конечно-разностных уравнений

$$
\Delta_{h} u=\frac{1}{h^{2}}\left(u_{l_{1}+1, l_{2} \ldots, l_{m}}+u_{l_{1}-1, l_{2} \ldots, l_{m}}+\ldots+u_{l_{1}, l_{2}, \ldots, l_{m}+1}+u_{l_{1}, l_{2}, \ldots, l_{m}-1}-2 m u_{l_{1}, l_{2}, \ldots, l_{m}}\right),
$$

являющихся наиболее употребимой конечно-разностной аппроксимацией лапласиана в пространстве $E^{m}$. Трудоемкость вычисления рассматриваемых функций приблизительно одинакова. 
9.2. Моделирование оттаивания мерзлых грунтов. Первый этап продолжается до появления межфазной границы. На этом этапе решается уравнение теплопроводности вида

$$
\rho_{1} c_{1} \frac{\partial u_{1}}{\partial t}=\lambda_{1} \frac{\partial^{2} u_{1}}{\partial x^{2}}+Q_{1}(x, t), \quad t>0, \quad 0<x<h,
$$

с начальным условием

$$
u_{1}=u_{0}(x), \quad t=0
$$

и граничными условиями

$$
\frac{\partial u_{1}}{\partial x}=k\left(u_{1}-u_{\text {air }}\right), \quad x=0 ; \quad \frac{\partial u_{1}}{\partial x}=0, \quad x=h,
$$

где $c_{1}$ - удельная теплоемкость, $\rho_{1}$ - плотность, $\lambda_{1}$ - теплопроводность, $u_{1}$ - температура грунта (все параметры относятся к мерзлому грунту); $t$-время, $x$ - координата, $Q_{1}$ - мощность внешнего источника тепла.

Второй этап начинается при появлении межфазной границы (т.е. при начале оттаивания грунта на поверхности). Плавление продолжается до заданной глубины $L$. На втором этапе решается система уравнений теплопроводности с учетом фазового перехода вида

$$
\begin{array}{ll}
\rho_{1} c_{1} \frac{\partial u_{1}}{\partial t}=\lambda_{1} \frac{\partial^{2} u_{1}}{\partial x^{2}}+Q_{1}(x, t) ; & \eta(t)<x<h, \\
\rho_{2} c_{2} \frac{\partial u_{2}}{\partial t}=\lambda_{2} \frac{\partial^{2} u_{2}}{\partial x^{2}}+Q_{2}(x, t) ; & 0<x<\eta(t),
\end{array}
$$

с начальным условием $u_{2}=u_{f}, u_{1}=u_{1,0}$ и граничными условиями

$$
\begin{gathered}
\frac{\partial u_{2}}{\partial x}=k\left(u_{2}-u_{\text {air }}\right), \quad x=0 ; \\
\lambda_{1} \frac{\partial u_{1}}{\partial x}-\lambda_{2} \frac{\partial u_{2}}{\partial x}=\rho_{1} r_{\text {melt }} \frac{d \eta(t)}{d t}, \quad x=0 ; \\
u_{1}=u_{2}=u_{f}, \quad x=\eta(t) ; \\
\frac{\partial u_{1}}{\partial x}=0, \quad x=h ;
\end{gathered}
$$

где $Q=A \cdot \exp (-2 a x)$, априорно неизвестная функция $x=\eta(t)$ определяет положение границы оттаивания и находится в процессе решения системы дифференциальных уравнений.

9.2.1. Решение задачи методом интегральных уравнений. Характеристики талого грунта (см. [7] и http://minkor.ru/upload/spravochnik/190609-2.pdf - онлайн-справочник по теплофизическим свойствам грунтов):

$$
\begin{array}{ll}
f=0,02, & \rho=1800 \frac{\mathrm{K} \Gamma}{\mathrm{M}^{3}}, \quad \lambda=0,47 \frac{\mathrm{BT}}{\mathrm{M} \cdot \mathrm{K}}, \quad c=778 \frac{\text { Дж }}{\mathrm{K \Gamma} \cdot \mathrm{K}} \\
f=0,12, & \rho=1800 \frac{\mathrm{K} \Gamma}{\mathrm{M}^{3}}, \quad \lambda=0,85 \frac{\mathrm{BT}}{\mathrm{M} \cdot \mathrm{K}}, \quad c=1278 \frac{\text { Дж}}{\mathrm{K} \cdot \mathrm{K}} .
\end{array}
$$

Характеристики мерзлого грунта:

$$
\begin{aligned}
& f=0,03, \quad \rho=1700 \frac{\mathrm{K} \Gamma}{\mathrm{M}^{3}}, \quad \lambda=0,56 \frac{\mathrm{BT}}{\mathrm{M} \cdot \mathrm{K}}, \quad c=750 \frac{\text { Дж }}{\mathrm{K \Gamma} \cdot \mathrm{K}} ; \\
& f=0,12, \quad \rho=1800 \frac{\mathrm{K} \Gamma}{\mathrm{M}^{3}}, \quad \lambda=0,78 \frac{\mathrm{BT}}{\mathrm{M} \cdot \mathrm{K}}, \quad c=1222 \frac{\text { Дж }}{\mathrm{K \Gamma} \cdot \mathrm{K}} .
\end{aligned}
$$

Таким образом, погрешность усредненных значений практически пренебрежима. Среднемассовую теплоту таяния грунта считаем равной $r_{\text {melt }}=335000 f$. 
9.2.2. Оттаивание грунта при помощи поверхностного нагрева. Рассмотрим случай, когда на поверхности значение температуры задано и равно $u_{m}$, на бесконечной глубине значение температуры равно $u_{0}$, такое же значение имеет начальная температура грунта. Выражение для температурного поля согласно описанному в статье методу имеет следующий вид:

$$
u(x, t)=H(x, t)-\frac{r_{\text {melt }}}{c} \int_{0}^{\infty} \frac{\exp \left[-\frac{\left(x-x_{N}\right)^{2}}{4 a\left(t-t_{f}\left(x_{N}\right)\right)}\right]-\exp \left[-\frac{\left(x+x_{N}\right)^{2}}{4 a\left(t-t_{f}\left(x_{N}\right)\right)}\right]}{2 \sqrt{\pi a\left(t-t_{f}\left(x_{N}\right)\right)}} 1\left(t-t_{f}\left(x_{N}\right)\right) d x_{N},
$$

где

$$
H(x, t)=\left(u_{m}-u_{0}\right) \operatorname{erfc}\left(\frac{x}{2 \sqrt{a t}}\right)+u_{0}
$$

Интегральное уравнение (15) для рассматриваемого случая принимает вид

$$
u_{f}=H\left(x, t_{f}(x)\right)-\frac{r_{\text {melt }}}{c} \int_{0}^{x} \frac{\exp \left[-\frac{\left(x-x_{N}\right)^{2}}{4 a\left(t_{f}(x)-t_{f}\left(x_{N}\right)\right)}\right]-\exp \left[-\frac{\left(x+x_{N}\right)^{2}}{4 a\left(t_{f}(x)-t_{f}\left(x_{N}\right)\right)}\right]}{2 \sqrt{\pi a\left(t_{f}(x)-t_{f}\left(x_{N}\right)\right)}} d x_{N}, \quad x>0 .
$$

Полученное интегральное уравнение решается аналитически. Решение отыскивается в виде $t_{f}(x)=k x^{2}$, где $k$ - неизвестный постоянный параметр. Введем обозначение $p=1 /(4 a k)$; тогда интегральное уравнение приводится после подстановки $t_{f}(x)=x^{2} /(4 a p)$ к нелинейному уравнению

$$
u_{f}=\left(u_{m}-u_{0}\right) \operatorname{erfc}(\sqrt{p})+u_{0}-\frac{2 r_{\text {melt }}}{c \sqrt{p / \pi}} \int_{0}^{\pi / 4}\left(\exp \left(-p \operatorname{tg}^{2} x\right)-\exp \left(-p \operatorname{ctg}^{2} x\right)\right) d x .
$$

Рассматриваемое нелинейное уравнение после отделения корня успешно решается методом хорд и касательных.

9.2.3. Результаты расчетов. При влажности $12 \%$ и плотности грунта (как мерзлого, так и талого) $\rho=1800 \mathrm{\kappa} \Gamma / \mathrm{M}^{3}$

$$
\lambda_{1}=0,78 \frac{\mathrm{BT}}{\mathrm{M} \cdot \mathrm{K}}, \quad c_{1}=1222 \frac{\text { Дж }}{\mathrm{K} \Gamma \cdot \mathrm{K}}, \quad \lambda_{2}=0,85 \frac{\mathrm{BT}}{\mathrm{M} \cdot \mathrm{K}}, \quad c_{2}=1278 \frac{\text { Дж }}{\mathrm{K} \cdot \mathrm{K}} ;
$$

соответствующие средние значения

$$
\lambda=0,815 \frac{\mathrm{BT}}{\mathrm{M} \cdot \mathrm{K}}, \quad c=1250 \frac{\text { Дж }}{\mathrm{K} \Gamma \cdot \mathrm{K}}
$$

(погрешность примерно 4\%). Значения температур в градусах Цельсия: $u_{0}=-10 ; u_{m}=2000$; $u_{f}=0$. Вычисленные значения параметров: при невязке уравнения $10^{-7}: k=249664$ и $p=2,764$. Для оттаивания грунта до глубины в 1 м потребуется 69,35 час. Для сравнения, если не учитывать скрытую теплоту таяния (т.е. при $r_{\text {melt }}=0$ ): при невязке уравнения $4,5 \cdot 10^{-7}$ имеем $k=174985$ и $p=3,944$. Для оттаивания грунта до глубины в 1 м потребовалось бы 48,61 час.

9.2.4. Сравнение численного решения методом интегральных уравнений и точного аналитического решений. Имеем

$$
\begin{gathered}
\frac{r_{\text {melt }}}{c} \sum_{j=1}^{i} x_{i j}\left(t_{i}-t_{j}\right)=H_{i}\left(t_{i}\right)-u_{f}, \quad i=1, \ldots, N ; \\
H_{i}(t)=\left(u_{m}-u_{0}\right) \operatorname{erfc}\left(\frac{x_{i}}{2 \sqrt{a t}}\right)+u_{0}, \\
x_{i j}(t) \approx \frac{1}{2}\left\{\operatorname{erf} \frac{h(i-j+1 / 2)}{2 \sqrt{a t}}-\operatorname{erf} \frac{h(i-j-1 / 2)}{2 \sqrt{a t}}-\operatorname{erf} \frac{h(i+j+1 / 2)}{2 \sqrt{a t}}+\operatorname{erf} \frac{h(i+j-1 / 2)}{2 \sqrt{a t}}\right\}
\end{gathered}
$$


Таблица 1. Результаты вычислений.

\begin{tabular}{|c|c|c|}
\hline$x_{i}, \mathrm{~cm}$ & $t_{i}$, час & $t_{f}\left(x_{i}\right)$, час \\
\hline 4,5 & 0,14 & 0,14 \\
\hline 9,5 & 0,63 & 0,63 \\
\hline 19,5 & 1,46 & 1,46 \\
\hline 24,5 & 2,63 & 2,64 \\
\hline 29,5 & 4,16 & 4,16 \\
\hline 34,5 & 8,25 & 8,25 \\
\hline 39,5 & 10,81 & 10,82 \\
\hline 44,5 & 13,72 & 13,73 \\
\hline 49,5 & 16,98 & 16,99 \\
\hline 54,5 & 20,58 & 20,60 \\
\hline
\end{tabular}

\begin{tabular}{|l|c|c|}
\hline$x_{i}, \mathrm{~cm}$ & $t_{i}$, час & $t_{f}\left(x_{i}\right)$, час \\
\hline 59,5 & 24,53 & 24,55 \\
\hline 64,5 & 28,83 & 28,85 \\
\hline 69,5 & 33,48 & 33,50 \\
\hline 74,5 & 38,47 & 38,49 \\
\hline 79,5 & 43,80 & 43,83 \\
\hline 84,5 & 49,49 & 49,52 \\
\hline 89,5 & 55,52 & 55,55 \\
\hline 94,5 & 61,90 & 61,93 \\
\hline 99,5 & 68,62 & 68,66 \\
\hline
\end{tabular}

при $i \neq j$. Выражение сингулярного слагаемого $(i=j)$ :

$$
x_{i i}(t) \approx \operatorname{erf}\left(\sqrt{\frac{h}{8 a k}}\right)-\frac{B e^{-B^{2}}}{(2 i-3 / 2) \sqrt{\pi}}+B^{2} \operatorname{erfc}(B), \quad B=\left(2 i-\frac{3}{2}\right) \sqrt{\frac{h}{2 a k}} ;
$$

с учетом равенства $k \approx\left(t_{i}-t_{i-1}\right) / h$ имеем

$$
B=\left(2 i-\frac{3}{2}\right) \frac{h}{\sqrt{2 a\left(t_{i}-t_{i-1}\right)}} .
$$

В таблице 1 представлены значения времен таяния на различной глубине. Шаг по координате равен $1 \mathrm{~cm}, x_{i}$ - координаты фронта фазового перехода, $t_{i}$ - результаты расчета методом интегральных уравнений, $t_{f}\left(x_{i}\right)$ - точные значения времен плавления.

Данные таблицы 1 показывают высокую точность рассматриваемого численного метода. Затраты машинного времени составляют порядка 1 с на 1 шаг по координате. Примечательная особенность рассматриваемых задач - даже значительное увеличение шага интегрирования не приводит к существенному росту погрешности.

9.2.5. Оттаивание грунта при помощи СВЧ нагрева. Плотность мощности тепловыделения на единицу объема при СВЧ нагреве диэлектрика равна $Q=\omega \operatorname{Im} \varepsilon_{r} \varepsilon_{0} E^{2}$, где $\omega$ - угловая частота ЭМП, $\operatorname{Im} \varepsilon_{r}$ - мнимая часть комплексной относительной диэлектрической проницаемости диэлектрика, $\varepsilon_{0}$ - диэлектрическая постоянная, $E$ - напряженность электрического поля. Если проводимость $\gamma$ материала мала либо частота настолько велика, что $\gamma \ll \omega \varepsilon\left(\varepsilon=\operatorname{Im} \varepsilon_{r} \varepsilon_{0}\right)$, то диэлектрический нагрев является доминирующим механизмом передачи энергии ЭМП в вещество. Коэффициент, характеризующий глубину проникновения ЭМП в грунт, равен мнимой части волнового числа: $\alpha=\omega /\left(3 \cdot 10^{8}\right) \sqrt{\left(\left|\varepsilon_{r}\right|-\operatorname{Re} \varepsilon_{r}\right) / 2}$.

9.2.6. Краевые условия I рода. Характеристики грунта те же, что и в предыдущем пункте. Значения температур в градусах Цельсия: на поверхности $-u_{m}=10$; на большой глубине$u_{0}=-10$; температура фазового перехода $-u_{f}=0$. В таблице 2 представлены значения времен таяния на различной глубине. Шаг по координате равен 1 см, $x_{i}$ - координаты фронта фазового перехода, $t_{i}$ - результаты расчета методом интегральных уравнений. Исходные данные для расчета: вещественная часть относительной диэлектрической проницаемости мерзлого грунта $\operatorname{Re} \varepsilon_{r 1}=5,4$; вещественная часть относительной диэлектрической проницаемости талого грунта $\operatorname{Re} \varepsilon_{r 2}=\operatorname{Re} \varepsilon_{r 1}(1-f)+70 f$, мнимая часть относительной диэлектрической проницаемости мерзлого грунта $\operatorname{Im} \varepsilon_{r 1}=0,4$; мнимая часть относительной диэлектрической проницаемости талого грунта $\operatorname{Im} \varepsilon_{r 2}=\operatorname{Im} \varepsilon_{r 1}(1-f)+15 f$, циклическая частота $\omega=2 \pi \cdot 915 \cdot 10^{6}$, суммарный коэффициент поглощения ЭМП $b=\alpha_{1}+\alpha_{2}$, амплитуда напряженности электрического поля: $E_{m}=2 \cdot 10^{3} \mathrm{~B} /$ м. 


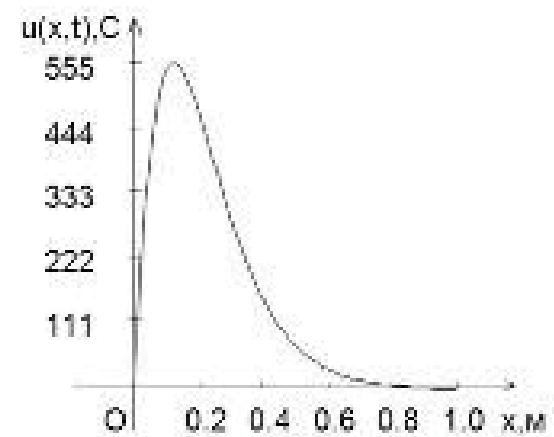

Рис. 1. График функции $H(x, t)$ при $t=10$ час.

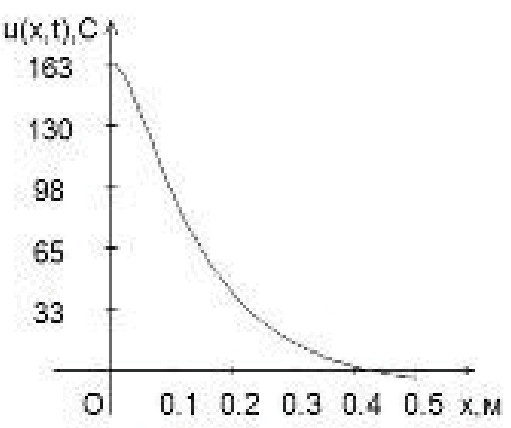

Рис. 2. График функции $H(x, t)$ при $t=1$ час.

Таблица 2. Результаты вычислений.

\begin{tabular}{|l|l|l|l|l|l|l|l|l|l|l|}
\hline$x_{i}$, см & 0,05 & 0,10 & 0,15 & 0,20 & 0,25 & 0,30 & 0,35 & 0,40 & 0,45 & 0,50 \\
\hline$t_{i}$, час & 0,27 & 0,40 & 0,56 & 0,79 & 1,12 & 1,57 & 2,20 & 3,04 & 4,16 & 5,63 \\
\hline
\end{tabular}

9.2.7. Температурное распределение при нулевой теплоте таяния. Находится посредством решения краевой задачи

$$
\rho c H_{t}=\lambda H_{x x}+A e^{-b x}, \quad x>0 ; \quad H(0, t)=u_{m}, \quad H(x, 0)=u_{0},
$$

где свободный член описывает диэлектрический нагрев при помощи СВЧ.

Решение краевой задачи находится аналитически и имеет вид

$$
H(x, t)=\left(u_{m}-u_{0}\right) \operatorname{erfc}\left(\frac{x}{2 \sqrt{a t}}\right)+u_{0}+\frac{A e^{-b x}}{\lambda b^{2}}\left(e^{a b^{2} t}-1\right)-\frac{A}{\rho c} \int_{0}^{t} \operatorname{erfc}\left(\frac{x}{2 \sqrt{a \tau}}\right) e^{a b^{2}(t-\tau)} d \tau,
$$

где $a=\lambda /(\rho c)$. На рис. 1 показан график рассматриваемого распределения температур.

9.2.8. Результаты расчетов. В таблице 2 представлены значения времен таяния на различной глубине. Шаг по координате равен 1 см, $x_{i}$ - координаты фронта фазового перехода, $t_{i}$ - результаты расчета описанным методом интегральных уравнений.

9.2.9. Краевые условия II рода. Характеристики грунта и исходные данные для расчета те же, что и в предыдущем пункте. Значения градиента температур на поверхности и на большой глубине нулевые: $\partial u / \partial x=0$. Начальное распределение: $u(x, 0)=u_{0}, x>0$. Решение соответствующей краевой задачи при нулевой теплоте фазового перехода находится аналитически и имеет вид

$$
H(x, t)=u_{0}+\frac{A e^{-b x}}{\left(\lambda b^{2}\right)\left(e^{a b^{2} t}-1\right)}-\frac{A}{\rho c b} \int_{0}^{t} \frac{e^{-x^{2} /(4 a \tau)}}{\sqrt{\pi a \tau}\left(e^{a b^{2}(t-\tau)}-1\right)} d \tau, \quad a=\frac{\lambda}{\rho c} .
$$

На рис. 2 показан график рассматриваемого распределения температур.

9.2.10. Результаты расчетов. В таблице 3 представлены значения времен таяния на различной глубине. Шаг по координате равен $1 \mathrm{~cm}, x_{i}$ - координаты фронта фазового перехода, $t_{i}$ - результаты расчета методом интегральных уравнений.

9.3. Двумерная задача. Рассмотрим задачу оттаивания плоского слоя грунта с теми же характеристиками. Оттаивание осуществляется при помощи иглы со спиралью накала, обеспечивающей 
Таблица 3. Результаты вычислений.

\begin{tabular}{|c|c|}
\hline$x_{i}$, см & $t_{i}$, час \\
\hline 0,02 & 0,15 \\
\hline 0,03 & 0,21 \\
\hline 0,04 & 0,25 \\
\hline 0,05 & 0,28 \\
\hline 0,06 & 0,31 \\
\hline 0,07 & 0,33 \\
\hline 0,08 & 0,35 \\
\hline
\end{tabular}

\begin{tabular}{|c|c|}
\hline$x_{i}, \mathrm{~cm}$ & $t_{i}$, час \\
\hline 0,09 & 0,37 \\
\hline 0,10 & 0,40 \\
\hline 0,11 & 0,42 \\
\hline 0,12 & 0,45 \\
\hline 0,13 & 0,48 \\
\hline 0,14 & 0,52 \\
\hline 0,15 & 0,56 \\
\hline
\end{tabular}

\begin{tabular}{|c|c|}
\hline$x_{i}, \mathrm{cм}$ & $t_{i}$, час \\
\hline 0,16 & 0,60 \\
\hline 0,17 & 0,64 \\
\hline 0,18 & 0,69 \\
\hline 0,19 & 0,74 \\
\hline 0,20 & 0,79 \\
\hline 0,25 & 1,12 \\
\hline 0,30 & 1,57 \\
\hline
\end{tabular}

\begin{tabular}{|c|c|}
\hline$x_{i}, \mathrm{~cm}$ & $t_{i}$, час \\
\hline 0,35 & 2,20 \\
\hline 0,40 & 3,04 \\
\hline 0,45 & 4,16 \\
\hline 0,50 & 5,63 \\
\hline
\end{tabular}

разогрев равномерно по толщине слоя. Решение соответствующей краевой задачи при нулевой теплоте фазового перехода находится аналитически и согласно (32)-(33) имеет вид

$$
H(M, t)=\int_{E^{2}} u_{0}(N) G_{0}(M, N, t) d V_{N}+\int_{0}^{t} \int_{E^{2}} \frac{P_{0} \delta\left(x_{N}, y_{N}\right)}{\rho c} G_{0}(M, N, t-\tau) d V_{N} d \tau,
$$

где

$$
G_{0}(M, N, t)=\frac{1}{4 \pi a t} \exp \left[-\frac{\left(x_{M}-x_{N}\right)^{2}+\left(y_{M}-y_{N}\right)^{2}}{4 a t}\right], \quad a=\frac{\lambda}{\rho c},
$$

$\delta\left(x_{N}, y_{N}\right)$ - двумерная дельта-функция, $P_{0}$ - мощность нагревательного элемента на единицу толщины слоя. Если начальное распределение температур постоянно, $u_{0}(N)=u_{0}=$ const, то функция $H(x, y, t)$ имеет вид

$$
H(x, y, t)=u_{0}+\frac{P_{0}}{4 \pi \lambda} E_{1}\left(\frac{x^{2}+y^{2}}{4 a t}\right)
$$

где $E_{1}(x)$ - интегральная экспоненциальная функция. Компоненты матрицы $X[t]$ равны

$$
\begin{gathered}
x_{k_{1} k_{2}}^{l_{1} l_{2}}(t)=u_{k_{1}}^{l_{1}}(t) u_{k_{2}}^{l_{2}}(t), \\
u_{k}^{l}(t)=\frac{1}{2}\left\{\operatorname{erf}\left(\frac{h(k-l+1 / 2)}{2 \sqrt{a t}}\right)-\operatorname{erf}\left(\frac{h(k-l-1 / 2)}{2 \sqrt{a t}}\right)\right\} .
\end{gathered}
$$

Расчет осуществляется при помощи решения системы (22), (29).

9.3.1. Вычисление сингулярного слагаемого в (22) и (29). Рассмотрим с этой целью интеграл

$$
J=\int_{V_{i}} \frac{\exp \left\{-\frac{\left(x-x_{i}\right)^{2}+\left(y-y_{i}\right)^{2}}{4 a\left(t_{x}\left(x-x_{i}\right)+t_{y}\left(y-y_{i}\right)\right)}\right\}}{\left[4 \pi a\left(t_{x}\left(x-x_{i}\right)+t_{y}\left(y-y_{i}\right)\right)\right] 1\left(t_{x}\left(x-x_{i}\right)+t_{y}\left(y-y_{i}\right)\right)} d x d y
$$

в котором функция $t_{f}(x, y)$ заменена на линейную часть ее ряда Тейлора с центром в точке $\left(x_{i}, y_{i}\right)$, $t_{x}=\partial t_{f} / \partial x\left(x_{i}, y_{i}\right), t_{y}=\partial t_{f} / \partial y\left(x_{i}, y_{i}\right),(x, y) \in V_{i}$. Предполагая, что ячейка является квадратом с центром в точке $\left(x_{i}, y_{i}\right)$, находим:

$$
J=\int_{-h / 2}^{h / 2} \int_{-h / 2}^{h / 2} \frac{\exp \left\{-\frac{x^{2}+y^{2}}{4 a\left(t_{x} x+t_{y} y\right)}\right\}}{4 \pi a\left(t_{x} x+t_{y} y\right)} 1\left(t_{x} x+t_{y} y\right) d x d y
$$


Разобьем интеграл на две части: $J=J_{0}+J_{1}$. Интеграл $J_{0}$ вычисляется по области круга, вписанного в ячейку (см. рис. 3):

$$
J_{0}=\int_{0}^{2 \pi} \int_{0}^{h / 2} \frac{\exp \left[-\frac{r}{4 a\left|\nabla t_{f}\right| \sin \varphi}\right]}{4 \pi a\left|\nabla t_{f}\right| \sin \varphi} 1(\sin \varphi) d x d y=\frac{2}{\pi} \int_{0}^{\pi / 2}\left\{1-\exp \left[-\frac{h}{8 a\left|\nabla t_{f}\right| \sin \varphi}\right]\right\} d \varphi,
$$

где $\left|\nabla t_{f}\right|=\sqrt{t_{x}^{2}+t_{y}^{2}}$. Этот круг делится на две части прямой $t_{x} x+t_{y} y=0$ (см. рис. 3 ). Вклад в интеграл дает только закрашенная половина круга, в которой $t_{x} x+t_{y} y>0$. Интеграл $J_{1}$ вычисляется по области ячейки вне круга. Эта часть также дает вклад в общий интеграл, так как в этой области также $t_{x} x+t_{y} y>0$. Форма соответствующей области сложна для точного вычисления. Используем тот факт, что участки данной области относительно близко прилегают к соответствующей полуокружности. Для вычисления интеграла $J_{1}$ используем приближенную квадратурную формулу

$$
J_{1} \approx\left(\frac{h^{2}}{2}-\frac{\pi h^{2}}{8}\right)\left\langle G\left(M_{i}, N, t_{x}\left(x-x_{i}\right)+t_{y}\left(y-y_{i}\right)\right)\right\rangle,
$$

где $\langle G\rangle$-среднее значение функции $G$ на соответствующей полуокружности, а сомножитель перед выражением в угловых скобках - площадь рассматриваемой области. В результате выражение интеграла $J_{1}$ примет вид

$$
J_{1} \approx\left(\frac{1}{2}-\frac{\pi}{8}\right) \frac{h}{\pi^{2} a\left|\nabla t_{f}\right|} \int_{0}^{\pi / 2} \frac{\exp \left[-\frac{h}{8 a\left|\nabla t_{f}\right| \sin \varphi}\right]}{\sin \varphi} d \varphi .
$$

Полученные одномерные интегралы эффективно вычисляются по формуле трапеций.

9.3.2. Аналитическое решение задачи Стефана. С учетом осевой симметрии данных задачи интегральное уравнение (15) в рассматриваемом двумерном случае упрощается и имеет вид

$$
u_{f}=H\left(r, t_{f}(r)\right)-\frac{r_{\mathrm{melt}}}{c} \int_{0}^{r} \frac{I_{0}\left(\frac{r r_{N}}{2 a\left(t_{f}(r)-t_{f}\left(r_{N}\right)\right)}\right)}{2 a\left(t_{f}(r)-t_{f}\left(r_{N}\right)\right)} r_{N} d r_{N}
$$

где

$$
H(r, t)=u_{0}+\frac{P_{0}}{4 \pi \lambda} E_{1}\left(\frac{r^{2}}{4 a t}\right),
$$

$I_{0}(x)$ - модифицированная формула Бесселя первого рода. Решение интегрального уравнения ищем в виде $t_{f}(r)=k r^{2}$, где $k$ - неизвестная константа. После подстановки этого выражения в интегральное уравнение и упрощений получаем уравнение относительно параметра $k$ :

$$
u_{f}=H(1, k)-\frac{\rho r_{\text {melt }}}{2 \lambda k} \int_{0}^{1} I_{0}\left[\frac{x}{2 a k\left(1-x^{2}\right)}\right] \exp \left[-\frac{1+x^{2}}{4 a k\left(1-x^{2}\right)}\right] \frac{x d x}{\left(1-x^{2}\right)},
$$

которое успешно решается элементарными методами хорд и касательных. Найденное точное аналитическое решение целесообразно использовать в качестве контрольного варианта для проверки вычислительных качеств вычислительного метода (29).

9.3.3. Результаты расчетов. Вычисления проводились при шаге $10 \mathrm{~cm}, P_{0}=100 \mathrm{~B}$ т $/ u_{0}=-5^{\circ} \mathrm{C}$. Погрешность численного решения изменяется приблизительно периодическим образом с увеличением области таяния. На рис. 4 показаны области таяния, вычисленные численным методом (множество узлов коллокаций) и при помощи точного аналитического решения (окружности).

Градиент решения, требуемый для вычисления сингулярных слагаемых, вычислялся при помощи квадратичной аппроксимации решения по сеточной области таяния методом наименьших квадратов. Определенную проблему представляют вычисления на начальном этапе по причине особенности точного решения (скорость фазового перехода стремится к бесконечности при $t \rightarrow 0$ ). 


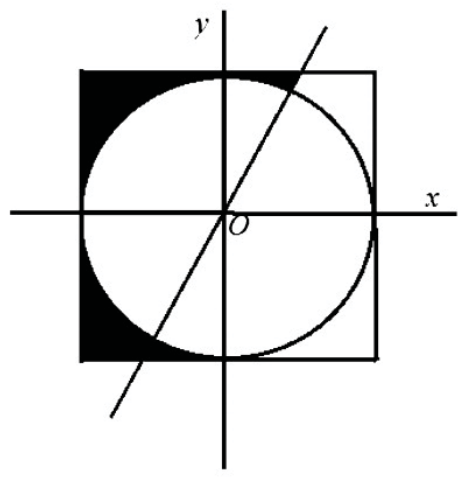

Рис. 3. Разбиение ячейки на области.
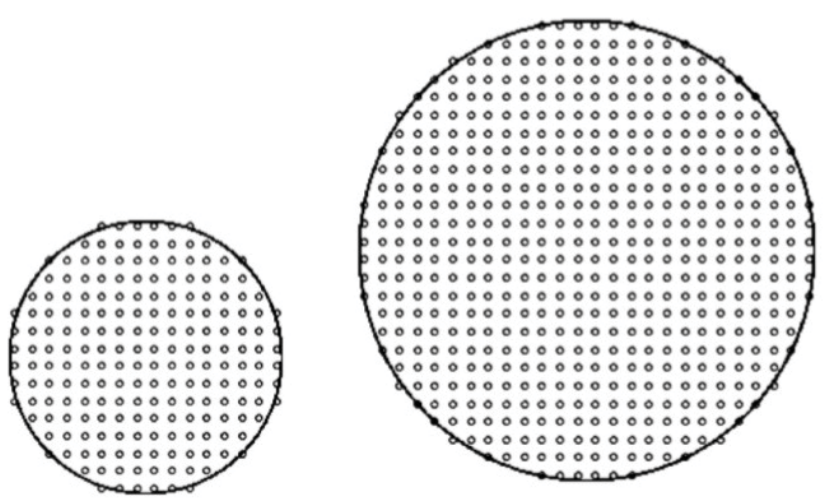

Рис. 4. Численное и аналитическое решение задачи Стефана (188 и 540 точек).

Таблица 4. Численное и точное решения.

\begin{tabular}{|c|c|c|c|}
\hline Количество точек & Численное решение & Точное решение & Погрешность, \% \\
\hline 188 & 23417,90 & 24266,80 & 3,5 \\
\hline 240 & 29967,62 & 30903,88 & 3,0 \\
\hline 540 & 6886078,64 & 6989668,75 & 1,5 \\
\hline
\end{tabular}

Точность численного решения улучшается, если использовать по возможности более точное начальное приближение на первых шагах интегрирования (порядка 50 точек).

10. Вывод. Предложен новый численный метод решения задачи Стефана, основанный на редукции исходной краевой задачи к эквивалентному нелинейному интегральному уравнению минимальной размерности. Особенностью метода является использование функции времени фазового перехода в качестве основной неизвестной функции. Данная параметризация является эффективным вычислительным приемом, позволяющим значительно упростить метод решения.

\section{СПИСОК ЛИТЕРАТУРЫ}

1. Анфиногентов В. И. Математическое моделирование СВЧ нагрева диэлектриков/ Дисс. на соиск. уч. степ. докт. техн. наук. - Казань, 2006.

2. Будак Б. М., Соловъева Е. Н., Успенский А. Б. Разностный метод со сглаживанием коэффициентов для решения задачи Стефана// Ж. вычисл. мат. мат. физ. - 1965. - 5, № 5. - С. 828-840.

3. Данилюк И. И. О задаче Стефана// Усп. мат. наук. - 1985. - 40, № 5 (245). - С. 133-185.

4. Каменомостская С. Л. Проблема Стефана// Мат. сб. - 1961. - 53, № 4. - С. 211-223.

5. Лыков А. В. Тепломассообмен. - М.: Энергия, 1971.

6. Мейрманов А. М. Задача Стефана. - Новосибирск: Наука, 1986.

7. Рожин И. И. Численное моделирование переходных процессов в прикладных задачах теплопроводности с фазовыми превращениями/ Дисс. на соиск. уч. степ. канд. физ.-мат. наук. - Якутск, 2005.

8. Рубинштейн Л. И. К вопросу о численном решении интегральных уравнений задачи Стефана// Изв. вузов. Мат. - 1958. - № 4. - С. 202-214.

9. Самарский А. А., Моисеенко Б. Д. Экономичная схема сквозного счета для многомерной задачи Стефана// Ж. вычисл. мат. мат. физ. - 1965. - 5, № 5. - С. 816-827. 
10. Dauzhenka T. A., Gishkeluk I. A. Quasilinear heat equation in three dimensions and Stefan problem in permafrost soils in the frame of alternating directions finite difference scheme// Proc. World Congress on Engineering (London, July 3-5, 2013). — London, 2013. - P. 1-6.

Арутюнян Роберт Владимирович

Московский государственный технический университет им. Н. Э. Баумана

(национальный исследовательский университет)

E-mail: rob57@mail.ru 